\title{
SPECIAL CYCLES AND AUTOMORPHIC FORMS ON ARITHMETICALLY DEFINED HYPERBOLIC 3-MANIFOLDS*
}

\author{
JOACHIM SCHWERMER ${ }^{\dagger}$
}

To the memory of Armand Borel

Introduction. An orientable hyperbolic 3-manifold is isometric to the quotient of hyperbolic 3-space $H^{3}$ by a discrete torsion free subgroup $\Gamma$ of the group $I s o\left(H^{3}\right)^{0}$ of orientation - preserving isometries of $H^{3}$. The latter group is isomorphic to the (connected) group $P G L_{2}(\mathbb{C})$, the real Lie group $S L_{2}(\mathbb{C})$ modulo its center $\{ \pm 1\}$. Generally, a discrete subgroup of $P G L_{2}(\mathbb{C})$ is called a Kleinian group. The group $\Gamma$ is said to have finite covolume if $H^{3} / \Gamma$ has finite volume, and is said to be cocompact if $H^{3} / \Gamma$ is compact.

Among hyperbolic 3-manifolds, the ones originating with arithmetically defined Kleinian groups form a class of special interest. Such an arithmetically defined 3manifold $H^{3} / \Gamma$ is essentially determined (up to commensurability) by an algebraic number field $k$ with exactly one complex place, an arbitrary (but possibly empty) set of real places and a quaternion algebra $D$ over $k$ which ramifies (at least) at all real places of $k$. These arithmetic Kleinian groups fall naturally into two classes. They can be distinguished by the compactness or non-compactness of the corresponding manifold $H^{3} / \Gamma$, since it turns out that this quotient always has finite volume.

If the arithmetic group $\Gamma$ is not cocompact in $P G L_{2}(\mathbb{C})$, then the defining field $k$ is an imaginary quadratic extension field $\mathbb{Q}(\sqrt{d}), d<0, d$ a square free integer. An arithmetic group of this type is commensurable to the group $P G L_{2}\left(\mathcal{O}_{d}\right)$ where $\mathcal{O}_{d}$ denotes the ring of integers in $k$. As early as $1892 \mathrm{~L}$. Bianchi studied this class of groups, today named after him.

If the arithmetic group $\Gamma$ is cocompact in $P G L_{2}(\mathbb{C})$, then the group $\Gamma$ arises from an order in a division quaternion algebra $D$ over $k$ which ramifies (at least) at all real places of $k$.

Within Thurston's geometrization program for 3-manifolds the class of hyperbolic 3-manifolds plays a fundamental role but is still not well understood. Due to the underlying connections with number theory the arithmetically defined hyperbolic 3 -manifolds seem to be in many ways more tractable. There is a fruitful interaction between geometric - topological, group - theoretical and arithmetic questions, methods and results. Many of the investigations carried through in recent years are dealt with in [9] or [31], both valuable sources.

Aside from the material covered in [31] there are some general geometric or arithmetic methods developed in the realm of the theory of arithmetic groups (in particular, those emerging in the theory of automorphic forms) which might help in understanding the specific case of arithmetically defined Kleinian groups. Thus, this paper is intended as an expository account of some of these investigations, putting results in

\footnotetext{
*Received April 23, 2004; accepted for publication July 27, 2004.

$\dagger$ Institute for Mathematics, University of Vienna, Nordbergstrasse 15, A-1090 Wien, Austria; The Erwin Schrödinger International Institute for Mathematical Physics, Boltzmanngasse 9, A-1090 Wien, Austria (joachim.schwermer@univie.ac.at).
} 
the appropriate general context. However, the focus will be strongly on arithmetically defined hyperbolic 3-manifolds.

In section 1, some familiar material on this class of manifolds is reviewed.

In section 2 we will discuss the concept of special cycles on arithmetic locally symmetric manifolds of the form $X / \Gamma$ (e. g. hyperbolic $n$-manifolds $H^{n} / \Gamma$ ). These special cycles arise naturally as connected components of the fixed point set of a morphism on $X / \Gamma$ induced by a rational automorphism of finite order on the underlying algebraic group. In particular, the rigorous use of non-abelian Galois cohomology serves as a suitable general framework to analyze the role these special cycles play.

This approach has the following applications in our context, discussed in section $3:$

- if $\Gamma$ is a Bianchi group, a study of the involution on $H^{3} / \Gamma$ induced by the non-trivial Galois automorphism of $k / \mathbb{Q}$ shows that these non-compact manifolds admit an abundance of totally geodesic hypersurfaces. They play a fundamental role in constructing non-bounding cycles, as well as in related questions in cohomology and its interpretation in terms of the automorphic spectrum (e.g. see [16], [17], [44])

- by use of the general formula for the intersection number of special cycles phrased in terms of non-abelian Galois cohomology (as proved in [45]) we discuss a slightly alternative approach to the non-vanishing result of Millson-Raghunathan [35] for the Betti numbers of certain compact arithmetically defined hyperbolic nmanifolds. These correspond (up to commensurability) to groups of units of nondegenerate quadratic forms on $l^{n+1}$ of index $(n, 1)$, all of whose conjugates are positive definite, and where $l \neq \mathbb{Q}$ is a totally real number field.

In the case $n=3$, this construction determines a specific class $\mathcal{M}$ of cocompact Kleinian groups under the exceptional isomorphism

$$
P G L_{2}(\mathbb{C}) \stackrel{\sim}{\rightarrow} \operatorname{Iso}\left(H^{3}\right)^{0} \tilde{\leftarrow} S O_{0}(3,1) .
$$

It is described in 1.6. There are some constraints on the defining field and the division algebra $D$ respectively.

In section 4, we will discuss how the principle of Langlands functoriality in the theory of automorphic forms makes it possible to obtain specific types of cuspidal automorphic forms on $G L_{2} / k$ where $k$ is an algebraic number field subject to certain conditions. This is a consequence of the base change lift as constructed in [26], [23]. This construction allows us (cf. [25]) to exhibit non-vanishing cuspidal cohomology classes for arithmetic subgroups (defined by congruence conditions) in $P G L_{2}(k)$ (up to subgroups of finite index) for the field in question. This result applies, in particular, to Bianchi groups.

Via the Jacquet-Langlands correspondence [22] between cuspidal automorphic representations for $G L_{2}$ and automorphic representations of its inner forms one obtains non-vanishing cohomology classes in cases of cocompact Kleinian groups. The class of groups dealt with contains the class $\mathcal{M}$ of cocompact Kleinian groups alluded to above but is much larger. Thus, this approach makes it even possible to construct non-vanishing cohomology classes on compact arithmetically defined hyperbolic 3manifolds which do not admit totally geodesic hypersurfaces, that is, in cases not covered by the geometric methods discussed in section 3 .

For example, this construction of non-vanishing automorphic cohomology classes can be carried out in the case of the collection $\mathcal{H}$ of cocompact arithmetic Kleinian 
groups which are commensurable to groups of units of skew-Hermitian forms on quaternionic vector spaces. This latter result was also obtained by Li-Millson [27] using theta series. However, the method [25] as discussed here gives a unified approach to the non-vanishing results in the case of the two classes $\mathcal{M}$ and $\mathcal{H}$. In both cases the arithmetically defined hyperbolic 3 -manifolds $H^{3} / \Gamma$ are determined by a quaternion division algebra $D$ over a field $k$ as above where $k$ contains a subfield of index 2. This subfield has to be a totally real field. Thus, a simple base change construction permits to exhibit non-trivial automorphic classes.

We illustrate the other results made possible in the automorphic framework by the following

ThEOREM. Let $H^{3} / \Gamma$ be an arithmetically defined hyperbolic 3-manifold where $\Gamma$ is a congruence group. Suppose that the defining field $k$ is a cubic non-normal extension of $\mathbb{Q}$. Then there exists a finite covering of $H^{3} / \Gamma$ with non-vanishing first Betti number.

Specific examples are given in 4.8 .

The various results exhibited in this paper give strong evidence that the virtual Haken conjecture (or, in its stronger form, known as the virtual positive Betti number conjecture) is true for arithmetically defined hyperbolic 3-manifolds. We discuss these consequences in 4.9 .

Nonetheless, the conjecture still remains open in a number of cases. For example, let $k=\mathbb{Q}(x)$ where $x$ is a root of the quintic polynomial $g=X^{5}-9 X+3$. The extension $k / \mathbb{Q}$ has degree 5 and it is non-normal. Let $D$ be a quaternion division algebra over $k$ which ramifies at least at the three real places (and one finite place) of $k$. Given an arithmetic Kleinian group originating with an order in $D$ the conjecture is not known to be true in this case.

Implicit in our discussion in this account is the hope that the ideas described here might help in gaining a better understanding of the geometry as well as the number theory of arithmetically defined hyperbolic 3-manifolds.

Author's work supported in part by FWF Austrian Science Fund, grant number P16762-N04.

Notation. (1) Let $F$ be an arbitrary finite extension of the field $\mathbb{Q}$, and denote by $\mathcal{O}_{F}$ its ring of integers. The set of places will be denoted by $V$, while $V_{\infty}$ (resp. $V_{f}$ ) will refer to the set of archimedean (resp. non-archimedean) places of $F$. The completion of $F$ at a place $v \in V$ is denoted by $F_{v}$, and its ring of integers by $\mathcal{O}_{v}\left(v \in V_{f}\right)$. For a given place $v \in V$ the normalized absolute value $\|_{v}$ on $F_{v}$ is defined as usual: if $v \in V_{\infty}$ is a real place we let $\|_{v}$ be the absolute value; if $v \in V_{\infty}$ is a complex place we put $\left|x_{v}\right|_{v}=x_{v} \cdot \bar{x}_{v}$; and if $v \in V_{f}$ is a finite place we put $\left|x_{v}\right|_{v}=N_{v}^{-\operatorname{ord}_{v}\left(x_{v}\right)}$, where $N_{v}$ denotes the cardinality of the residue field at the place $v$.

If the extension $F / \mathbb{Q}$ has degree $d$ one has $d=r_{1}+2 r_{2}$ where $r_{1}$ (resp. $r_{2}$ ) denotes the number of real (resp. complex) places of $F$.

We denote by $\mathbb{A}_{F}$ (resp. $\mathbb{I}_{F}$ ) the ring of adèles (resp. the group of idèles) of $F$. If $F=\mathbb{Q}$ the subscript will be omitted from the notation. There is the usual decomposition of $\mathbb{A}_{F}$ (resp. $\mathbb{I}_{F}$ ) into the infinite and finite part $\mathbb{A}_{F}=\mathbb{A}_{F, \infty} \times \mathbb{A}_{F, f}$ (resp. $\mathbb{I}_{F}=\mathbb{I}_{F, \infty} \times \mathbb{I}_{F, f}$ ).

(2) The algebraic groups we consider will be linear groups. If $H$ is an algebraic 
group defined over a field $k$, and $k^{\prime}$ is a commutative $k$-algebra, we denote by $H\left(k^{\prime}\right)$ the group of $k^{\prime}$-valued points of $H$. When $k^{\prime}$ is a field we denote by $H / k^{\prime}$ the $k^{\prime}$ algebraic group $H \times{ }_{k} k^{\prime}$ obtained from $H$ by extending the ground field from $k$ to $k^{\prime}$.

1. Arithmetically defined Kleinian groups. In this section, we review the basic definitions and aspects of arithmetically defined hyperbolic 3-manifolds and Kleinian groups. For more details, see for instance [9], [31], [41]. The theory of quaternion algebras over an algebraic number field is, as well as local questions, dealt with in [55], [42], [59], [51].

1.1. Kleinian groups. Hyperbolic 3-space is characterized as the unique 3dimensional connected, simply connected Riemannian manifold with constant sectional curvature -1 . One of the possible models of $H^{3}$ is given as the symmetric space attached to the real Lie group $S L_{2}(\mathbb{C})$, that is, $H^{3}=S U(2) \backslash S L_{2}(\mathbb{C})$. An orientable hyperbolic 3-manifold $M$ is isometric to the quotient of hyperbolic 3-space $H^{3}$ by a discrete torsion free subgroup $\Gamma$ of the group $I s o\left(H^{3}\right)^{0}$ of orientation-preserving isometries of $H^{3}$. The latter group is isomorphic to the (connected) group $P G L_{2}(\mathbb{C})$, the Lie group $S L_{2}(\mathbb{C})$ modulo its center $\{ \pm I\}$.

A Kleinian group $\Gamma$ is a discrete subgroup of $P G L_{2}(\mathbb{C})$ which acts properly discontinuously on $H^{3}$, i. e., for any given compact subset $C$ of $H^{3}$ the set $\{\gamma \in \Gamma$ । $\gamma C \cap C \neq \emptyset\}$ is finite. Thus, the stabilizer of a point in $H^{3}$ is finite. In particular, if $\Gamma$ is torsion free, it acts freely. Thus $M$ is of the from $H^{3} / \Gamma$ where $\Gamma$ is a torsion free Kleinian group. The group $\Gamma$ is said to have finite covolume if $H^{3} / \Gamma$ has finite volume, and is said to be cocompact if $H^{3} / \Gamma$ is compact. If the Kleinian group $\Gamma$ has torsion, the corresponding quotient space $H^{3} / \Gamma$ is an orbifold, that is, it locally looks like the quotient of a Euclidean space by a finite group. However, if $\Gamma$ is a finitely generated Kleinian group then $\Gamma$ has a torsion free subgroup of finite index (see e. g. [1]). Arithmetically defined Kleinian groups to be defined shortly form a class of finite covolume groups.

1.2. $\mathbf{k}$-forms of $\mathbf{P G L}_{2}$. In order to describe arithmetically defined Kleinian groups we shall consider $k$-forms of $P G L_{2}$ (or $S L_{2}$ ) over an algebraic number field. By definition, a linear algebraic group $G$ defined over $k$ is a $k$-form of the $k$-group $P G L_{2}$ (or $S L_{2}$ ) if there exists a field extension $k^{\prime} / k$ such that $G$ is isomorphic as a $k^{\prime}$-group to $P G L_{2} / k^{\prime}$ (or $S L_{2} / k^{\prime}$ ).

The $k$-forms in question can be described in the following way. Let $A$ be a quaternion algebra over the field $k$, that is, $A$ is a central simple algebra over $k$ of degree 2. Notice that $\operatorname{dim}_{k} A=4$. Let $G L(A)$ be the algebraic group defined over $k$ whose rational points over an extension $k^{\prime} / k$ equal the group of invertible elements in the $k^{\prime}$-algebra $A \otimes_{k} k^{\prime}$. The reduced norm defines a surjective homomorphism

$$
N r d: G L(A) \rightarrow G_{m}
$$

of $G L(A)$ into the multiplicative group over $k$. The kernel of the morphism $N r d$ is a semisimple, simply connected algebraic group over $k$, to be denoted $S L_{1}(A)$. This group is a $k$-form of the group $S L_{2} / k$.

The $k$-group $G L(A)$ has a one-dimensional center, and its derived group is $S L_{1}(A)$. Then the quotient $G$ of $G L(A)$ by its center is a $k$-form of $P G L_{2} / k$. This construction exhausts all possible $k$-forms of $P G L_{2} / k$.

1.3. Arithmetically defined Kleinian groups. Let $H$ be a reductive algebraic 
group defined over $k$. Then a subgroup $\Gamma$ of $H(k)$ is arithmetic if for an embedding $\varrho: H \rightarrow G L_{N}$ the group $\varrho(\Gamma)$ is commensurable with $\rho(H) \cap G L_{N}\left(\mathcal{O}_{k}\right)$, that is, the intersection is of finite index in both $\varrho(\Gamma)$ and $\varrho(H) \cap G L_{N}\left(\mathcal{O}_{k}\right)$. Notice that the latter group depends on the embedding only up to commensurability.

Specialised to discrete subgroups of $P G L_{2}(\mathbb{C})$ arithmeticity is as follows: Let $\Gamma$ be a discrete subgroup of $P G L_{2}(\mathbb{C})$. Then $\Gamma$ is said to be arithmetically defined if there exists an algebraic number field $k / \mathbb{Q}$ with exactly one complex place $w$ (that is, $r_{2}=1$ ), an arbitrary (but possibly empty) set $T$ of real places, a $k$-form $G$ of $P G L_{2} / k$ such that $G\left(k_{v}\right)$ is compact for $v \in T$ and an isomorphism

$$
P G L_{2}(\mathbb{C}) \stackrel{\sim}{\rightarrow} G\left(k_{w}\right), w \text { the complex place }
$$

which maps $\Gamma$ onto an arithmetic subgroup of $G(k)$ naturally embedded into $G\left(k_{w}\right)$. These arithmetically defined Kleinian groups fall naturally into two cases to be discussed separately later. They can be distinguished by the compactness or noncompactness of the corresponding hyperbolic manifold $H^{3} / \Gamma$, since it turns out that this quotient always has finite volume.

1.4. Bianchi groups. Here the arithmetic group $\Gamma$ is not cocompact in $P G L_{2}(\mathbb{C})$. Then $k$ is an imaginary quadratic extension of $\mathbb{Q}$, that is, $k$ is of the form $\mathbb{Q}(\sqrt{d}), d<0, d$ a square free integer. The $k$-form in question is just the group $P G L_{2}$ defined over $k$. An arithmetic group $\Gamma$ of this type is commensurable to the group $P G L_{2}\left(\mathcal{O}_{d}\right)$ where $\mathcal{O}_{d}$ denotes the ring of algebraic integers in $k=\mathbb{Q}(\sqrt{d})$. This ring forms a $\mathbb{Z}$-lattice in $\mathbb{C}$ with basis $1, \omega_{d}=\sqrt{d}$ with $\omega_{d}=d$ when $d \equiv 2,3 \bmod$ 4 and $\omega_{d}=(1 / 2)(1+\sqrt{d})$ when $d \equiv 1 \bmod 4$. Thus the groups $\Gamma_{d}:=P G L_{2}\left(\mathcal{O}_{d}\right)$ are discrete subgroups of $P G L_{2}(\mathbb{C})$. As early as $1892 \mathrm{~L}$. Bianchi studied this class of groups, today named after him. These groups and all its subgroups of finite index have finite covolume. Let $\mu(\Gamma)$ denote the volume of $H^{3} / \Gamma$ with respect to the hyperbolic metric. Following G. Humbert the value $\mu\left(\Gamma_{d}\right)$ can be expressed in terms of invariants only depending on the underlying field $k$ (see e. g. [13] or [4], Thm. 7.3.). More precisely, one has $\mu\left(\Gamma_{d}\right)=\left(\left|D_{k}\right|^{3 / 2} / \pi^{2}\right) \zeta_{k}(2)$ where $D_{k}$ denotes the discriminant of $k$ and $\zeta_{k}$ is the $\zeta$-function attached to $k$.

Following the work of Bianchi and Humbert [2], Swan [54] exhibited fundamental domains for the action of $\Gamma_{d}$ on $H^{3}$. A range of geometric or group-theoretical results as, for example, presentations for $\Gamma_{d}$ is based on this approach.

However, for an explicit computation of the cohomology of $\Gamma_{d}$ with integral coefficients this natural choice of a contractible space on which $\Gamma_{d}$ acts properly is inconvenient for the simple reason that the dimension of $H^{3}$ is three, whereas the virtual cohomological dimension of $\Gamma_{d}$ is two. This fact indicates that it may be possible for $\Gamma_{d}$ to act properly on a contractible space of dimension two. A space of this type was constructed by Mendoza [33] by use of Minkowski's reduction theory. He exhibits a $\Gamma_{d}$-invariant 2-dimensional deformation retract $I(k)$ of $H^{3}$ such that the quotient of $I(k)$ by any subgroup of $\Gamma_{d}$ of finite index is compact. The space $I(k)$ is endowed with a natural $C W$-structure such that the action of $\Gamma_{d}$ is cellular and the quotient $I(k) / \Gamma$ is a finite $C W$-complex. This construction opened the way for quite far reaching investigations regarding the cohomology of Bianchi groups and related questions in the theory of automorphic forms for these groups. In turn, the results obtained there had a direct impact on the geometric nature of the underlying manifold $H^{3} / \Gamma$. For these consequences see, for example, [50] [56]. 
1.5. Groups originating with orders in division algebras. Here the arithmetic group $\Gamma$ is cocompact in $P G L_{2}(\mathbb{C})$. Then the derived group of the $k$-form of $P G L_{2} / k$ in question is of the form $S L_{1}(D)$ where $D$ is a division quaternion algebra over $k$ which ramifies at all real places $v \in T$. Then the group $\Gamma$ originates with an order $\Lambda$ in $D$. By definition, an order $\Lambda$ in $D$ is a subring containing the unit element $1_{D}$ which is a finitely generated $\mathcal{O}_{k}$ module with $\mathcal{O}_{k} \Lambda=D$. The latter condition characterizes a full $\mathcal{O}_{k}$ lattice in $D$.

In order to discuss this case more thoroughly it is necessary to recall some results pertaining to quaternion algebras over number fields.

Let $F$ be an algebraic number field of degree $d=[F: \mathbb{Q}]$. Suppose that $F$ has $r_{1}$ real places and $r_{2}$ complex places so that $d=r_{1}+2 r_{2}$. The field $F$ embeds naturally in each local field $F_{v}$ corresponding to an element $v$ in the set $V$ of places of $F$.

Let $Q$ be a quaternion algebra over $F$, that is, $Q$ is a central simple $F$-algebra of degree two. Viewed as a vectorspace over $F$ the algebra $Q$ has a basis $1, i, j, k$ subject to the relations $i^{2}=a, j^{2}=b, i j=-j i=k$ for some elements $a, b \in F^{*}$. Although the quaternion algebra $Q$ does not uniquely determine the elements $a, b \in F^{*}$ we may also use the notation $Q=Q(a, b \mid F)$ emphasizing the choice of $a$ and $b$. Given a place $v \in V$ there is the local analogue

$$
Q_{v}=Q \otimes_{F} F_{v},
$$

defined as the tensor product over $F$ of $Q$ with $F_{v}$. The algebra $Q_{v}$ is a central simple algebra over $F_{v}$. If $v \in V_{\infty}$ is a complex place, that is, $F_{v}=\mathbb{C}$, this algebra is isomorphic to the matrix algebra $M_{2}(\mathbb{C})$. If $v \in V_{\infty}$ is a real place the algebra $Q_{v}$ over $\mathbb{R}$ is isomorphic either to the matrix algebra $M_{2}(\mathbb{R})$ or to the division algebra $\mathbb{H}=Q(-1,-1 \mid \mathbb{R})$ of Hamilton's quaternions. A similar dichotomy exists in the case of a non-archimedean place $v \in V_{f}$. For each local field $F_{v}, v \in V_{f}$, there is a unique quaternion division algebra $C_{v}$ over $F_{v}$. Using the unique unramified quadratic extension $F_{v}(\sqrt{a})$ where $a$ is a unit in the ring $\mathcal{O}_{v}$ of integers in $F_{v}$ it can be described as a cyclic algebra. Thus, the quaternion algebra $Q \otimes_{F} F_{v}$ is isomorphic either to the matrix algebra $M_{2}\left(F_{v}\right)$ or the unique division algebra $C_{v}$. This is a consequence of the fact that a quaternion algebra over an arbitrary field $L$ is isomorphic either to $M_{2}(L)$ or a division algebra.

We say that $Q$ ramifies at $v \in V$, or that $v$ is ramified in $Q$ if $Q \otimes_{F} F_{v}$ is isomorphic to a division algebra, otherwise $Q$ splits at $v \in V$. Hilbert's law of reciprocity implies that a quaternion algebra $Q$ over $F$ splits at all but a finite number of places. The set $\operatorname{Ram}(Q)=\{v \in V \mid Q$ ramifies at $v\}$ has even cardinality. Notice that the isomorphism class of $Q$ over $F$ is determined by its ramification set $\operatorname{Ram}(Q)$. Conversely, given a set $S \subset V \backslash\left\{v \in V_{\infty} \mid v\right.$ complex place $\}$ so that $S$ has even cardinality there exists a quaternion algebra over $F$ with ramification set equals $S$.

These results imply in the case at hand that for a given number field $k$ with exactly one complex place $w$ and an arbitrary non-empty set $T$ of real places there are infinitely many non-isomorphic quaternion algebras $D$ over $k$ such that $D$ ramifies at $v \in T$. This is achieved by supposing that the ramification set $\operatorname{Ram}(D)=\{v \in V \mid D$ ramifies at $v\}$ is any finite set of even cardinality so that $T \subset \operatorname{Ram}(D)$.

REMARK. The case of Bianchi groups can be subsumed under the previous approach in terms of quaternion algebras as well. Here the quaternion algebra is $Q=M_{2}(k), k$ an imaginary quadratic extension of $\mathbb{Q}$, and the choice of $Q$ is equivalent to the specification $\operatorname{Ram}(Q)=\emptyset$. 
1.6. Orthogonal groups. Hyperbolic 3-space can be realized in various models. One of these, the Lobachevski model, provides a useful interpretation of its group of isometries as an orthogonal group of a real quadratic space. More precisely, let $(V, q)$ be a 4-dimensional quadratic space over $\mathbb{R}$ with a quadratic form of index 1 , that is, by Sylvester's law of inertia $q$ has the form $x_{1}^{2}+x_{2}^{2}+x_{3}^{2}-x_{4}^{2}$ with respect to a suitable basis of $V$. Let $S=\left\{x \in V \mid q(x)<0, x_{4}>0\right\}$ be the positive cone in $V$, and let $P S$ be its projective quotient. This space is in one-to-one correspondence with one sheet of the hyperboloid defined by $\left\{x \in V \mid q(x)=-1, x_{4}>0\right\}$ and endowed with the Riemannian metric induced by the Lorentzian metric $d x_{1}^{2}+d x_{2}^{2}+d x_{3}^{2}-d x_{4}^{2}$. Thus, $P S$ carries a natural metric realizing hyperbolic 3-space. The full group of isometries of this model of $H^{3}$ is the projective quotient of the subgroup $\{g \in O(V, q) \mid g(S)=S\}$ of the orthogonal group $O(V)$ of the quadratic space $(V, q)$. This identification provides the exceptional isomorphism.

$$
P G L_{2}(\mathbb{C}) \stackrel{\sim}{\rightarrow} I s o\left(H^{3}\right)^{0} \tilde{\leftarrow} S O_{0}(3,1)
$$

of the group of orientation preserving isometries of $H^{3}$ with the identity component of the real Lie group $S O(3,1)$.

In our context, this isomorphism leads to investigating the algebraic group $H$ (defined over some number field $k$ ) underlying the Lie group, that is, whose group of real points is isomorphic to $S O(3,1)$. One then has the problem to determine the arithmetic subgroups in $H(k)$ giving rise to discrete subgroups in $S O_{0}(3,1)$ in this way.

For simplicity, we discuss this more generally for the group $S O(n, 1)$ but we mainly restrict ourselves to the case of cocompact arithmetically defined groups.

First, there is the natural choice of orthogonal groups (or groups of units) of quadratic forms over number fields. Let $k$ be a totally real algebraic number field of degree $[k: \mathbb{Q}]=m, f$ a non-degenerate quadratic form on $k^{n+1}$ and $G$ the special orthogonal group $S O(f)$ of $f$. Suppose that the form $f$ as a form over $k_{v}\left(v \in V_{\infty}\right)$ has index $(n, 1)$ at one archimedean place $v_{0} \in V_{\infty}$ of $k$ and is positive definite at all other archimedean places of $k$. Then the symmetric space $X$ associated with the group of real points is hyperbolic $n$-space. Let $\Gamma$ be a torsion free arithmetic subgroup of $S O(f)$. Then $\Gamma$ acts freely on $X$, and the quotient $X / \Gamma$ is a hyperbolic manifold of finite volume. If $k$ is not equal to the field $\mathbb{Q}$, the manifold $X / \Gamma$ is compact. Let $k=\mathbb{Q}$. For $n \geq 5$, every non-degenerate quadratic form on $\mathbb{R}^{n+1}$ which is indefinite over the real numbers non-trivially represents zero over its field of definition, hence $\Gamma$ is not cocompact. Both cases can occur for $n=2,3,4$, that is, there exist cocompact arithmetic groups $\Gamma$ as well as not cocompact ones.

In general, for $n$ odd, $S O(n, 1)$ has another type of cocompact arithmetically defined subgroups, commensurable to groups of units of skew hermitian forms in quaternionic vector spaces. If $n=7$, one should add arithmetic subgroups of the triality form of $\mathrm{SO}_{8}$ to those.

In the case of hyperbolic 3-manifolds all possible types of cocompact arithmetically defined subgroups in $P G L_{2}(\mathbb{C})$ are described in 1.5 . Let $k$ be a totally real number field, $k \neq \mathbb{Q}, f$ a non-degenerate quadratic form on $k^{4}$ such that for all $v$ not equal to a given $v_{0}$ in $V_{\infty}$ the form $f_{v}$ over $k_{v} \cong \mathbb{R}$ is positive definite while $f_{v_{0}}$ over $k_{v_{0}}$ has signature $(3,1)$, and let $\Gamma$ be an arithmetic subgroup in $S O(f)$. This class of cocompact Kleinian groups corresponds to the class of arithmetically defined subgroups in $S L_{1}(D), D$ a quaternion division algebra of the form $B \otimes_{k} k^{\prime}$, where 
$B$ is a quaternion algebra over the totally real field $k$ which ramifies at all places $v \neq v_{0}$ and $k^{\prime}$ is a quadratic externsion of $k$ with exactly one complex place (that is, $k^{\prime}=k(\sqrt{a}), a<0, a \in k, a$ square free) (see e. g. [31], Thm. 10.2.1).

2. Construction of cycles. In this section, $G$ is a connected algebraic group defined over $\mathbb{Q}$. Notice, if $H$ is an algebraic group defined over some number field $k$ then the group $\operatorname{Res}_{k / \mathbb{Q}} H$ obtained from $H$ by restriction of scalars from $k$ to $\mathbb{Q}$ is an algebraic $\mathbb{Q}$-group.

2.1. Non-abelian Galois cohomology. Let $\Theta$ be a group acting on a set $A$. The action of $s \in \Theta$ is written as $a \mapsto s a={ }^{s} a$, and we define $A^{\Theta}=\operatorname{Fix}(\Theta, A)=$ $\left\{a \in A \mid{ }^{s} a=a\right.$ for all $\left.s \in \Theta\right\}$. If $\Theta=\langle\tau\rangle$ is generated by one element $\tau$, we write $A^{\Theta}=A^{\tau}$.

If the group $\Theta$ acts on a group $A$ as a group of automorphisms, then $H^{1}(\Theta, A)$ denotes the first non abelian cohomology set of $\Theta$ in $A$. It is defined as follows (cf. [52], Chap. I,5): A cocycle of $\Theta$ in $A$ is a map $\gamma: \Theta \rightarrow A, s \mapsto \gamma_{s}$ so that $\gamma_{s t}=\gamma_{s}{ }^{s} \gamma_{t}$ for all $s, t \in \Theta$. One writes $\gamma=\left(\gamma_{s}\right)$. The set of all cocycles of $\Theta$ in $A$ will be denoted by $Z^{1}(\Theta, A)$. There is the trivial cocycle, defined by the constant map $\Theta \rightarrow A$, $s \mapsto 1_{A}$. Two cocycles $\gamma$ and $\zeta$ are said to be equivalent if there is an element $a \in A$ so that $\zeta_{s}=a^{-1} \gamma_{s}{ }^{s} a$ for all $s \in \Theta$. This notion defines an equivalence relation on $Z^{1}(\Theta, A)$. Then $H^{1}(\Theta, A)$ is the set of equivalence classes. It is a pointed set, and its distinguished element is the class of the trivial cocycle to be denoted by $1_{\Theta}$.

Suppose the groups $\Theta$ and $A$ act on a set $M$ in a compatible way, that is, we have ${ }^{s}(a m)={ }^{s} a^{s} m$ for all $s \in \Theta, a \in A, m \in M$. Then, given a cocycle $\gamma=\left(\gamma_{s}\right), s \in \Theta$, for $H^{1}(\Theta, A)$ there is a $\gamma$-twisted $\Theta$-action on $M$ given by $m \mapsto \gamma_{s}{ }^{s} m, s \in \Theta$. We denote the fixed points of this action by $M(\gamma), \gamma$ a given cocycle for $H^{1}(\Theta, A)$.

2.2. Fixed points of $\mathbb{Q}$-rational automorphisms of finite order. Let $\Theta$ be a finite abelian group of $\mathbb{Q}$-rational automorphisms of $G / \mathbb{Q}$. Choose a maximal compact subgroup $K$ of $G(\mathbb{R})$, stable under the group $\Theta$. Then the group $\Theta$ acts on the symmetric space $X=K \backslash G(\mathbb{R})$. Given a $\Theta$-stable torsion free arithmetic subgroup $\Gamma$ of $G(\mathbb{Q})$ there is a natural action of $\Theta$ on the locally symmetric space $X / \Gamma$. If $\gamma=\left(\gamma_{s}\right), s \in \Theta$, is a cocycle of $\Theta$ in $\Gamma$, there are $\gamma$-twisted actions of $\Theta$ on $G$ and on $\Gamma$, defined by $g \mapsto \gamma_{s}{ }^{s} g \gamma_{s}^{-1}, g \in G, s \in \Theta$. The induced action of $\Theta$ on $X$ is given by $x \mapsto{ }^{s} x \gamma_{s}^{-1}, x \in X, s \in \Theta$. The new action of $\Theta$ induced on $X / \Gamma$ coincides with the previous one. Let $\Gamma(\gamma)$ be the set of elements in $\Gamma$ fixed by the $\gamma$-twisted action of $\Theta$ and let $X(\gamma)$ be the set of fixed points of the $\gamma$-twisted $\Theta$-action on $X$. Then the natural map $\pi_{\gamma}: X(\gamma) / \Gamma(\gamma) \rightarrow X / \Gamma$ is injective. This can be seen as follows.

Let $x, y \in X(\gamma)$, suppose there exists $\delta \in \Gamma$ so that $x=y \cdot \delta$. Since $x$ and $y$ are fixed under the $\gamma$-twisted $\Theta$-action we have

$$
{ }^{s} x \cdot \gamma_{s}^{-1}=x \quad \text { resp. } \quad{ }^{s} y \cdot \gamma_{s}^{-1}=y \quad \text { for all } \quad s \in \Theta .
$$

This implies, by applying $s \in \Theta$ to the equation $x=y \cdot \delta$,

$$
\begin{gathered}
{ }^{s} x={ }^{s}(y \cdot \delta)={ }^{s} y{ }^{s} \delta \\
=y \cdot \gamma_{s}{ }^{s} \delta
\end{gathered}
$$

and hence

$$
x \gamma_{s}=y \cdot \gamma_{s}^{s} \delta,
$$


that is

$$
x=y \cdot \gamma_{s}^{s} \delta \gamma_{s}^{-1} .
$$

But the group $\Gamma$ acts freely on $X$ so that $\delta=\gamma_{s}{ }^{s} \delta \gamma_{s}^{-1}$ for all $s \in \Theta$. Thus, $\delta \in \Gamma(\gamma)$. The image of $\pi_{\gamma}$

$$
F(\gamma):=i m \pi_{\gamma} \cong X(\gamma) / \Gamma(\gamma)
$$

lies in the fixed point set $\operatorname{Fix}(\Theta, X / \Gamma)$. Notice that $F(\gamma)$ depends only on the class in $H^{1}(\Theta, \Gamma)$ represented by the cocycle $\gamma$. The spaces $F(\gamma)$ are non-empty since the action of $\Theta$ on $X$ is via isometries ([21] Thm. 13.5.). Any two points of $X(\gamma)$ are joined by a unique geodesic of $X$ ([21] Lemma 14.3.). Thus, $F(\gamma)$ is a connected totally geodesic closed submanifold of $X / \Gamma$. Its fundamental group is isomorphic to $\Gamma(\gamma)$.

All fixed points of $\Theta$ acting on $X / \Gamma$ arise by this construction. Consider a point $\bar{x} \in \operatorname{Fix}(\Theta, X / \Gamma)$ represented by $x \in X$. Then there exist uniquely determined elements $\gamma_{s} \in \Gamma$ such that ${ }^{s} x=x \gamma_{s}, s \in \Theta$. Then we have

$$
{ }^{s}\left({ }^{t} x\right)=x \gamma_{s t}
$$

respectively,

$$
{ }^{s}\left({ }^{t} x\right)={ }^{s}\left(x \gamma_{t}\right)={ }^{s} x^{s} \gamma_{t}=x \gamma_{s}{ }^{s} \gamma_{t}
$$

so that $\gamma_{s t}=\gamma_{s}{ }^{s} \gamma_{t}$ for all $s, t \in \Theta$. Hence $\gamma=\left(\gamma_{s}\right), s \in \Theta$, is a cocycle of $\Theta$ in $\Gamma$. Given another representative $x^{\prime}=x \cdot c, c \in \Gamma$, of $\bar{x}$ the attached cocycle is determined by $\gamma_{s}^{\prime}=c^{-1} \gamma_{s}{ }^{s} c, s \in \Theta$. Thus every point in the fixed point set Fix $(\Theta, \Gamma)$ determines a unique class in $H^{1}(\Theta, \Gamma)$. As a consequence, the fixed point set is a disjoint union of the connected non-empty sets $F(\gamma), \gamma \in H^{1}(\Theta, \Gamma)$, that is

$$
F i x(\Theta, X / \Gamma)=\coprod_{\gamma \in H^{1}(\Theta, \Gamma)} F(\gamma)
$$

2.3. Special cycles. Suppose that $\mu$ is a $\mathbb{Q}$-rational automorphism of $G / \mathbb{Q}$ of finite order. By 2.2., the set of fixed points of the induced morphism $\mu$ on $X / \Gamma$ decomposes as the disjoint union of connected non-empty sets, that is,

$$
F i x(\langle\mu\rangle, X / \Gamma)=\coprod_{H^{1}(\langle\mu\rangle, \Gamma)} F(\gamma) .
$$

The connected totally geodesic closed submanifolds $F(\gamma)$ are parametrized by the first non-abelian cohomology set $H^{1}(\langle\mu\rangle, \Gamma)$ of the group $\langle\mu\rangle$ (generated by $\mu$ ) in $\Gamma$. The component corresponding to the basepoint $1_{\mu}$ in $H^{1}(\langle\mu\rangle, \Gamma)$ will be called a special cycle, to be denoted $C(\mu, \Gamma)$.

Each of the components $F(\gamma)$ of $F i x(\langle\mu\rangle, X / \Gamma)$ can be viewed as a special cycle. Indeed, the component $F(\gamma)$ corresponds to the $\mathbb{Q}$-rational automorphism obtained by twisting $\mu$ with $\gamma$. 
By construction, the special cycle $C(\mu, \Gamma)$ is diffeomorphic to the locally symmetric space originating with the group of real points of the algebraic $\mathbb{Q}$-group $G(\mu)$ of fixed points under $\mu$. There is a diffeomorphism

$$
X\left(1_{\mu}\right) / \Gamma\left(1_{\mu}\right) \stackrel{\sim}{\rightarrow} C(\mu, \Gamma) .
$$

2.4. Orientability. In general, it is not the case that the closed submanifolds $C(\mu, \Gamma)$ in $X / \Gamma$ carry a natural orientation. However, in order to analyze the contribution of $C(\mu, \Gamma)$ (e.g. via its fundamental class) to the homology or cohomology of the quotient $X / \Gamma$ one has to deal with this problem. The question of orientability arises in an even stronger form if one actually wants to determine the intersection number (so defined) of a special cycle with a suitable submanifold $Y$ of complementary dimension in $X / \Gamma$. In such a case (e.g. if $Y$ is also given as a special cycle as it is often the case) one needs that all connected components of the intersection of these two cycles are orientable. As proved in [45] Prop. 2.2., this situation can always be achieved up to a subgroup of finite index in $\Gamma$. There is the following result of interest for us.

Proposition. Let $\Theta$ be a finite group of $\mathbb{Q}$-rational automorphisms of a reductive algebraic group $G$ defined over $\mathbb{Q}$. Suppose that $\Gamma \subset G(\mathbb{Q})$ is a $\Theta$-stable arithmetic subgroup of $G$. Then there exists a normal $\Theta$-stable torsion free arithmetic subgroup $\Gamma_{0}$ in $\Gamma$ such that $\Gamma_{0}(\gamma) \subset G(\gamma)(\mathbb{R})^{0}$ for all cocycles $\gamma$ in $H^{1}\left(\Theta, \Gamma_{0}\right)$, that is, the corresponding components $F(\gamma)$ of the fixed point set Fix $\left(\Theta, X / \Gamma_{0}\right)$ in $X / \Gamma_{0}$ are orientable.

REMARK. If $\Theta$ is the group generated by two automorphisms $\sigma, \tau$ of $G$ commuting with one another a cocycle $\gamma$ of $\Theta$ in $\Gamma$ uniquely determines two cocycles $\gamma_{\mu}$ for $H^{1}(\langle\mu\rangle, \Gamma), \mu=\sigma, \tau$. For the corresponding set of fixed points one has $X(\gamma)=$ $X\left(\gamma_{\sigma}\right) \cap X\left(\gamma_{\tau}\right)$. By passing to a suitable subgroup $\Gamma^{\prime} \subset \Gamma$ it can be achieved that for all cocycles $\gamma^{\prime}$ of $\Theta$ in $\Gamma^{\prime}$ the corresponding groups $\Gamma^{\prime}\left(\gamma^{\prime}\right)$ resp. $\Gamma^{\prime}\left(\gamma_{\mu}^{\prime}\right), \mu=\sigma, \tau$ act on $X\left(\gamma^{\prime}\right)$ resp. $X\left(\gamma_{\mu}^{\prime}\right)$ preserving orientations. This result ([45], Cor. 2.4) settles the question of orientability for two special cycles and the connected components of their intersection.

2.5. Examples. (1) Let $k=\mathbb{Q}(\sqrt{d}), d<0, d$ square-free, $d \in \mathbb{Z}$, be an imaginary quadratic number field and let $\sigma$ be the nontrivial Galois automorphism of the extension $k / \mathbb{Q}$. The group $\langle\sigma\rangle$ generated by $\sigma$ acts on the algebraic $\mathbb{Q}$-group $\operatorname{Res}_{k / \mathbb{Q}} P G L_{2}$ obtained by restriction of scalars from the $k$-group $P G L_{2}$. The arithmetic group $\Gamma_{d}=P G L_{2}\left(\mathcal{O}_{d}\right)$ is stable under this action. Given a torsion free $\sigma$-stable subgroup $\Gamma$ of finite index in $\Gamma_{d}$ the involution $\sigma$ induces an automorphism of order two on the hyperbolic 3-manifold $H^{3} / \Gamma$. By 2.3. the set of fixed points decomposes as a disjoint union of its connected components $F(\gamma)$ parametrized by the non-abelian Galois cohomology $H^{1}(\langle\sigma\rangle, \Gamma)$. This latter set is determined in [44] in the case $\Gamma=\Gamma_{d}$. One has to distinguish two types of spaces $X(\gamma)$ giving rise to connected components $F(\gamma)=X(\gamma) / \Gamma(\gamma)$. First, the base point $1_{\sigma}$ in $H^{1}\left(\langle\sigma\rangle, \Gamma_{d}\right)$ and the cocycle $\eta:\langle\sigma\rangle \rightarrow \Gamma_{d}$, uniquely determined by assigning $\sigma \mapsto\left(\begin{array}{cc}1 & \omega_{d} \\ 0 & 1\end{array}\right)$ respectively provide $2-$ dimensional subspaces in $X=H^{3}$ diffeomorphic to the hyperbolic plane $H^{2}$. Second, there are two isolated fixed points in $X$. As a result, a 2-dimensional component $F(\gamma)$ in $\operatorname{Fix}\left(\langle\sigma\rangle, H^{3} / \Gamma\right)$ is either a covering of $X\left(1_{\sigma}\right) / \Gamma_{d}\left(1_{\sigma}\right)$ or of $X(\eta) / \Gamma_{d}(\eta)$. The 
former one is homeomorphic to $H^{2} / P G L_{2}(\mathbb{Z})$ whereas $X(\eta) / \Gamma_{d}(\eta)$ is homeomorphic to the quotient of $H^{2}$ by the image of the group $\left\{\left(a_{i j}\right) \in S L_{2}(\mathbb{Z}) \mid a_{12} \equiv 0 \bmod 2\right\}$ in $P G L_{2}(\mathbb{Z})$. This is a surface whose Euler characteristic is zero. In addition, depending on the discriminant of $k$ one might find a finite number of isolated fixed points in Fix $\left(\langle\sigma\rangle, H^{3} / \Gamma\right)$. For a principal congruence subgroup

$$
\Gamma(\mathfrak{a}):=\left\{\alpha \in \Gamma_{d} \mid \alpha \equiv 1 \bmod \mathfrak{a}\right\}, \mathfrak{a} \text { an ideal in } \mathcal{O}_{d}
$$

there is a list in [43], Prop. 4.1., giving exactly the number of components of each of the above types. Of course, the quantity essentially depends on the data $\mathfrak{a}$, that is, the prime ideals occuring in $\mathfrak{a}$, and the discriminant of $k$.

(2) Let $G$ be a connected reductive $\mathbb{Q}$-group, let $\sigma, \tau$ two $\mathbb{Q}$-rational automorphisms of $G / \mathbb{Q}$ of finite order, and suppose that $\sigma$ and $\tau$ commute with one another. Choose a $\langle\sigma, \tau\rangle$-stable maximal compact subgroup $K$ of $G(\mathbb{R})$. Then the group $\langle\sigma, \tau\rangle$ acts on $X=K \backslash G(\mathbb{R})$. If $\Gamma$ is a $\langle\sigma, \tau\rangle$-stable torsion free arithmetic subgroup of $G(\mathbb{Q})$ there is an induced action of $\langle\sigma, \tau\rangle$ on $X / \Gamma$. This is the general framework considered in [45] in order to analyze the intersection number of special cycles attached to $\sigma$ and $\tau$.

This situation can be achieved, as exhibited in specific cases by MillsonRaghunathan [35], in the following way: Let $\sigma$ be an involution of $G / \mathbb{Q}$. Given a rational point $\bar{x}$ in the cycle $C\left(1_{\sigma}, \Gamma\right)$ represented by $x$ in $X\left(1_{\sigma}\right)$ there is the Cartan involution $\theta_{x}$ corresponding to $x$. Since $\bar{x}$ is rational $\theta_{x}$ is defined over $\mathbb{Q}$. Then $\tau:=\sigma \theta_{x}$ is a rational involution of $G$ as well, and $\sigma$ and $\tau$ commute with one another. The spaces $X\left(1_{\sigma}\right)$ and $X\left(1_{\tau}\right)$ are totally geodesic submanifolds of $X$ of complementary dimensions, meeting only at $x$. Then the corresponding special cycles $C(\sigma, \Gamma)$ and $C(\tau, \Gamma)$ have at least one isolated intersection point. There the two cocycles intersect transversally. By going over to a suitable subgroup $\Gamma^{\prime}$ of $\Gamma$ it may be achieved that the cycles $C\left(\sigma, \Gamma^{\prime}\right)$ and $C\left(\tau, \Gamma^{\prime}\right)$ resp. the connected components of their intersection are orientable (cf. 2.4.).

(3) Let $k \neq \mathbb{Q}$ be a totally real algebraic number field, $f$ a non-degenerate quadratic form on $k^{n+1}$ and $G=S O(f)$ the special orthogonal group of $f$. Suppose that $f$ as a form over $k_{v}\left(v \in V_{\infty}\right)$ has index $(n, 1)$ at one archimedean place $v_{0}$ and is positive definite at all other archimedean places. From our assumptions, a torsion free arithmetic subgroup $\Gamma$ of $S O(f)$ gives rise to a compact hyperbolic $n^{-}$ manifold $X / \Gamma$. We write $k^{n+1}$ as a direct sum of two orthogonal subspaces $W_{1}, W_{2}$. Let $f_{1}$ and $f_{2}$ be the restrictions of $f$ on $W_{1}$ and $W_{2}$ respectively and $H_{1}$ (resp. $H_{2}$ ) the special orthogonal group of $f_{1}$ (resp. $f_{2}$ ), identified with the subgroup of $G$ leaving $W_{2}$ (resp. $W_{1}$ ) pointwise fixed. These subgroups are the sets of fixed points of obvious involutions of $G$ commuting with one another.

For example, this approach provides the existence of a totally geodesic closed hyperbolic submanifold of codimension one in $H^{n} / \Gamma$ (cf. [34]). To see this write $f$ in diagonal form. We fix a coordinate hyperplane $Y$ such that the restriction $f^{\prime}$ of $f$ to $Y$ has index $(n-1,1)$ at $v_{0}$. Let $H$ be the subgroup of $G=S O(f)$ leaving the orthogonal complement of $Y$ in $k^{n+1}$ pointwise fixed. Its restriction to $Y$ is the special orthogonal group of $f^{\prime}$. It is the fixed point set of an involution $\sigma$, induced by the orthogonal transformation of $k^{n+1}$ which acts as the identity on $Y$ and by -1 on the complement of $Y$. Let $X_{H}$ be the symmetric space attached to $H(\mathbb{R})$. Then, as 
a result of 2.2., the corresponding arithmetic quotient

$$
X_{H} /(\Gamma \cap H(k)) \rightarrow X / \Gamma
$$

is a totally geodesic closed submanifold of $X / \Gamma$. By construction, its codimension is one.

REMARK. It is natural in the case $n=3$ to reinterprete these totally geodesic hypersurfaces in $H^{3} / \Gamma, \Gamma \subset S O(f)$ as above, in the framework of the corresponding class of arithmetic groups in $S L_{1}(D)$. Here $D$ is a quaternion division algebra of the form $B \otimes_{k} k^{\prime}$, where $B$ is a quaternion algebra over the totally real field $k$ which ramifies at all places $v \neq v_{0}$ and $k^{\prime}$ is a quadratic externsion of $k$ with exactly one complex place (that is, $k^{\prime}=k(\sqrt{a}), a<0, a \in k, a$ square free) (see e. g. [31], Thm. 10.2.1). Then these hypersurfaces come up as components of the fixed point set of the involution induced by the non-trivial Galois automorphism of the quadratic extension $k^{\prime} / k$.

\section{Special cycles and cohomology of arithmetic hyperbolic $n$-manifolds.}

3.1. Intersection numbers. Let $G$ be a connected reductive $\mathbb{Q}$-group endowed with a rational $\mathbb{Q}$-automorphism $\mu$ of finite order, and let $\Gamma \subset G(\mathbb{Q})$ be a $\langle\mu\rangle-$ stable torsion free arithmetic subgroup. By definition, the connected components of the fixed point set Fix $(\langle\mu\rangle, X / \Gamma)$ of the automorphism induced on $X / \Gamma$ are special cycles. These are totally geodesic closed submanifolds in $X / \Gamma$ of the form

$$
F(\gamma)=X(\gamma) / \Gamma(\gamma) \rightarrow X / \Gamma
$$

where $\gamma$ is a cocycle representing a class in $H^{1}(\langle\mu\rangle, \Gamma)$. We are interested in cases where a special cycle $Y$ is orientable and its fundamental class is not homologous to zero in $X / \Gamma$, in singular homology or homology with closed supports, as necessary. As pointed out in 2.4., by passing to a suitable subgroup of finite index in $\Gamma$ we may suppose that special cycles are orientable.

The usual way to go about the second question is to produce an orientable submanifold $Y^{\prime}$ of complementary dimension such that the intersection product (so defined) of its fundamental class with that of $Y^{\prime}$ is non-zero. In doing so, if $X / \Gamma$ is non-compact, one has to assume that one of $Y, Y^{\prime}$ is compact, while the other one is not. In order to find a non-zero intersection, if at all possible, it is often necessary to replace $\Gamma$ by a subgroup of finite index.

For the sake of convenience we recall the definition of the intersection number of two closed oriented submanifolds $Y, Y^{\prime}$ in an oriented manifold $M$ of dimension $\operatorname{dim} M=m$. Suppose that the intersection $Y \cap Y^{\prime}$ is compact and that the submanifolds have complementary dimensions, that is, $\operatorname{dim} Y+\operatorname{dim} Y^{\prime}=m$. Let $N=Y$ or $Y^{\prime}$, and let $U$ be a tubular neighborhood of $N$ in $M$. The normal bundle of $N$ in $M$ is oriented in a natural way. Hence there is a distinguished isomorphism

$$
j_{x}: H^{m-\operatorname{dim} N}\left(U_{x},(U-N)_{x} ; \mathbb{Z}\right) \rightarrow \mathbb{Z}
$$

where $U_{x}$ denotes the fibre of the normal bundle in $U$ over $x \in N$. Then there is a unique class $w_{N}$ in $H^{m-\operatorname{dim} N}(U, U-N ; \mathbb{Z})$ such that $w_{N}$ is mapped under the composite of maps

$$
H^{m-\operatorname{dim} N}(U, U-N ; \mathbb{Z}) \rightarrow H^{m-\operatorname{dim} N}\left(U_{x},(U-N)_{x} ; \mathbb{Z}\right) \rightarrow \mathbb{Z}
$$


to the element 1 in $\mathbb{Z}$ (cf. [36], Thm. 10.4). The intersection number of $Y$ and $Y^{\prime}$ is defined by

$$
[Y]\left[Y^{\prime}\right]=\left(w_{Y} \cup w_{Y^{\prime}}\right)[M]
$$

that is, by evaluating the cup product $w_{Y} \cup w_{Y^{\prime}}$, in $H_{c}^{m}(M, \mathbb{Z})$ on the fundamental class $[M]$ of $M$.

There are various implementations of the general scheme just outlined. We discuss one of these in the context of arithmetically defined hyperbolic $n$-manifolds. For other examples we refer to [49].

3.2. The Betti numbers of compact hyperbolic $n$-manifolds. In this section we consider the case of a compact arithmetically defined hyperbolic $n$-manifold as constructed in 1.6., resp. 2.5. More precisely, $k \neq \mathbb{Q}$ is a totally real number field, $f$ denotes a nondegenerate quadratic form on $k^{n+1}$, and let $G$ be the group $S O(f)$. Suppose that there is exactly one place $v_{0} \in V_{\infty}$ so that $f$ over $k_{v_{0}}$ has index $(n, 1)$ and is positive definite at all other archimedean places of $k$. If $\Gamma$ is a group of units in $S O(f)$, that is, a torsion free arithmetic subgroup $\Gamma \subset G(k)$ the quotient space $X / \Gamma$ is a compact orientable hyperbolic $n$-manifold. Given a natural number $j, 1 \leq j<n$, this manifold is naturally equipped with a pair of rational involutions $\sigma, \tau$ commuting with one another so that the corresponding special cycles $C\left(1_{\sigma}, \Gamma\right)$ and $C\left(1_{\tau}, \Gamma\right)$ have (complementary) dimensions $j$ and $n-j$ respectively. The underlying symmetric spaces $X\left(1_{\sigma}\right)$ and $X\left(1_{\tau}\right)$ intersect in exactly one point because the group of real points of the fixed points of $\langle\sigma, \tau\rangle$ acting on $G$ is compact. This is a consequence of the compactness criterion (cf. [3], §8). By arranging the orientations of $X, X\left(1_{\sigma}\right)$ and $X\left(1_{\tau}\right)$ suitably it can be achieved that the intersection number in this point is positive. Thus, by the general analysis of the intersection number of special cycles carried through in [45], one obtains the following result: There exists a $\langle\sigma, \tau\rangle$-stable subgroup $\Gamma^{\prime}$ of finite index in $\Gamma$ such that

$$
\left[C\left(1_{\sigma}, \Gamma^{\prime}\right)\right]\left[C\left(1_{\tau}, \Gamma^{\prime}\right)\right]=\sum \chi(F(\gamma))
$$

where the sum ranges over the elements $\gamma$ in the kernel of the natural restriction map

$$
H^{1}\left(\langle\sigma, \tau\rangle, \Gamma^{\prime}\right) \rightarrow H^{1}\left(\langle\sigma\rangle, \Gamma^{\prime}\right) \times H^{1}\left(\langle\tau\rangle, \Gamma^{\prime}\right),
$$

and where all Euler characteristics $\chi(F(\gamma))$ of the corresponding connected components of the intersection $C\left(1_{\sigma}, \Gamma^{\prime}\right) \cap C\left(1_{\tau}, \Gamma^{\prime}\right)$ are positive. Hence, the cycles contribute non-trivially to cohomology. As a consequence, one obtains by Poincar duality a nonvanishing result for the Betti numbers $b_{j}, j=1, \ldots, n-1$, of the compact hyperbolic $n$-manifold $H^{n} / \Gamma^{\prime}$. We have the assertion first proved in [35]:

3.3. Theorem. Let $k \neq \mathbb{Q}$ be a totally real algebraic number field, let $f$ be a non-degenerate quadratic form on $k^{n+1}$ of index $(n, 1)$, all of whose conjugates are positive definite. Let $\Gamma$ be the group of units of $f$ that is, $\Gamma$ is an arithmetic subgroup of the special orthogonal group $S O(f)$ of $f$. Then there exists a torsion free subgroup $\Gamma^{\prime}$ of finite index in $\Gamma$ such that all Betti numbers $b_{j}, j=1, \ldots, n-1$, of the compact oriented hyperbolic $n$-manifold $H^{n} / \Gamma^{\prime}$ are non-zero.

3.4. Remark. The motivation for looking at intersections of cycles has its origin in Millson-Ragunathans's work. The starting point for the approach here is the 
formula [45] Thm. 4.11, for the intersection number of special cycles phrased in terms of non-abelian Galois cohomology. The method is general not only with respect to the ambient group but also the arithmetic group. Hence, this approach applies to the groups of units of certain quadratic or hermitian forms, defined over suitable algebraic number fields, which do not represent zero rationally over their field of definition as well as to other more general situations not dealt with in [35]. In particular, the rigorous use of non-abelian Galois cohomology serves as a suitable general framework to analyze the connected components of the intersection of special cycles and the questions of orientability involved.

3.5. The first Betti number. The hyperbolic manifolds dealt with so far in this section admit totally geodesic closed submanifolds. Their very existence made the construction of non-bounding cycles possible. If one is only interested in the first Betti number of hyperbolic $n$-manifolds this geometric approach, originally initiated by Millson [34] has been strengthened in [30] by taking in a simple result in geometric group theory. This method applies first to the cases of 3.3. and also to the nonarithmetic lattices in $S O(n, 1)$ constructed in [12]. However, it is a critical assumption that a totally geodesic submanifold of codimension one in $H^{n} / \Gamma$ is available. The result of Lubotzky is

TheOREm. Let $M$ be an oriented $n$-dimensional hyperbolic manifold of finite volume. Suppose that $M$ contains a totally geodesic submanifold $F$ of codimension one. Then $\Gamma:=\pi_{1}(M)$ has a subgroup of finite index which admits a homomorphism onto a virtually non abelian free group.

To give an idea of the proof consider the case where the submanifold $F$ separates $M$, i. e., divides the latter into two parts with common boundary $F$. Let $A$ and $B$ be their fundamental groups and $C=\pi_{1}(F)$. Then $\Gamma$ is the free product of $A$ and $B$ with amalgamated subgroup $C$. The group $C$ is of infinite index in $A$ and $B$. Using Borel's density theorem one constructs a homomorphism $\pi: \Gamma \rightarrow S$ onto a finite group such that $\pi(C) \varsubsetneqq \pi(A), \pi(C) \varsubsetneqq \pi(B)$. Consequently, $\Gamma$ is mapped onto the free product of $\pi(A)$ and $\pi(B)$ with amalgamated subgroup $\pi(C)$. As an amalgam of finite groups it is virtually free. A further group theoretical analysis of this situation, using [53] (cf. p. 123, Exerc. 3) implies the result.

The case where $F$ does not separate $M$ is analogous, with the free product replaced by an $H N N$ construction. More precisely, one considers the fundamental group of the open manifold obtained by cutting $M$ along $F$.

Remark. The result in [30] has been generalized by Margulis and Vinberg to a much larger family of groups [32]. It is now a result in geometric group theory.

3.6. Bianchi groups. In this subsection we discuss some specific results pertaining to the first Betti number of an arithmetically defined non-compact hyperbolic 3 -manifold. It is of the form $H^{3} / \Gamma$ where $\Gamma$ is a torsion free arithmetic subgroup in $\Gamma_{d}=P G L_{2}\left(\mathcal{O}_{d}\right), \mathcal{O}_{d}$ the ring of integers in an imaginary quadratic number field $k=\mathbb{Q}(\sqrt{d})$.

(1) It follows from reduction theory that there exists a compact manifold $M \subset$ $H^{3} / \Gamma$ with boundary $\partial M$ such that inclusion $M \rightarrow H^{3} / \Gamma$ is a homotopy equivalence ([3] Theorem 17.10 or [20]). The boundary is a disjoint union of finitely many tori corresponding to the $\Gamma$-conjugacy classes of Borel subgroups in $P G L_{2} / k$. Consider, 
in rational cohomology, the segment

$$
H^{1}(M) \stackrel{r^{1}}{\longrightarrow} H^{1}(\partial M) \rightarrow H_{c}^{2}(M)
$$

of the long exact sequence of the pair $(M, \partial M)$. The image of the restriction map $r^{1}$ to the cohomology of the boundary has dimension equal $(1 / 2) \operatorname{dim} H^{1}(\partial M)$, that is, equal to the number $c$ of components in $\partial M$. [This is a consequence of a general result on $I m r^{m}$ proved by using duality for compact manifolds with boundary of dimension $\operatorname{dim} M=2 m+1$ cf. [7], VIII, proof of 9.6.]. This implies $b_{1}\left(H^{3} / \Gamma\right) \geq c$.

(2) The fact that the Bianchi groups $\Gamma_{d}$ have a subgroup of finite index with a non abelian free quotient was first proved in [15] using a blend of geometric investigations within a fundamental domain for $\Gamma_{d}$ and some number theoretical results. However, given a congruence subgroup $\Gamma(\mathfrak{p}), \mathfrak{p}=p \mathcal{O}_{d}, p$ a prime which does not split in $\mathcal{O}_{d}$, the method in [30] allows one to determine a lower bound for the rank of the free quotient of $\Gamma(\mathfrak{p})$ in question.

(3) As discussed in 2.5. (1) the non-compact manifolds $H^{3} / \Gamma$ admit an abundance of totally geodesic hypersurfaces. They arise as 2-dimensional components $F(\gamma)$ of the set of fixed points under the involution induced by the non-trivial Galois automorphism of the underlying extension $k / \mathbb{Q}$. Each of these special cycles leads to a decomposition of $\Gamma$ either as a free product $A * B$ with amalgamated subgroup $\pi_{1}(F(\gamma))$ or a $H N N$ construction over $\pi_{1}(F(\gamma))$.

\section{Automorphic forms and some non-vanishing results.}

4.1. Automorphic spectrum and cohomology. Let $G$ be a connected semi simple algebraic group defined over an algebraic number field $k$. Suppose that the $k$-rank of $G$ is greater than zero. An arithmetic torsion free subgroup $\Gamma$ of $G(k)$ acts properly and freely on the symmetric space $X$ associated to the group of real points of $G$. The quotient $X / \Gamma$ is a non-compact complete Riemannian manifold of finite volume. Let $(v, E)$ be a finite dimensional representation of $G(\mathbb{R})$ on a complex vector space $E$. The cohomology groups $H^{*}(X / \Gamma, E)$ (computed e. g. via the de Rham complex of $E$-valued differential forms on $X / \Gamma$ ) can be interpreted in terms of the automorphic spectrum of $\Gamma$. With this framework in place, there is a sum decomposition of the cohomology into the cuspidal cohomology $H_{c u s p}^{*}(X / \Gamma, E)$ (i. e. classes represented by cuspidal automorphic forms for $\Gamma$ ) and the so called Eisenstein cohomology constructed as the span of appropriate residues or derivatives of Eisenstein series attached to cuspidal automorphic forms on the Levi components of proper parabolic $k$-subgroups of $G$ (see [10], [11] and [29]).

This is the final step in a program which began with the work of Harder in the case $S L_{2} / k$ [17], [19] or, more general, groups of $\mathbb{Q}-$ rank one [18]. By use of Langlands' theory of Eisenstein series, Harder constructed in this case a subspace in $H^{*}(X / \Gamma, E)$ (a complement to the image of the cohomology with compact supports) whose elements are obtained either by taking suitable values of Eisenstein series, or residues of such. As a result, there is a sum decomposition of the cohomology of $\Gamma$ (recall that the $k$-rank of $G$ is one)

$$
H^{*}(X / \Gamma, E)=H_{c u s p}^{*}(X / \Gamma, E) \otimes H_{E i s}^{*}(X / \Gamma, E)
$$

where the Eisenstein cohomology carries the contribution "at infinity" corresponding to the $\Gamma$-conjugacy classes of (minimal!) parabolic $k$-subgroups. 
The cuspidal cohomology is defined analytically (cf. e. g. [5]) and can be described in terms of the cuspidal spectrum in the space $L^{2}(G(\mathbb{R}) / \Gamma)$ of complex valued square integrable functions on $G(\mathbb{R}) / \Gamma$, viewed as usual as a unitary $G(\mathbb{R})$-module via left translations. Hence it is contained in the space of cohomology classes which can be represented by square-integrable forms.

The cuspidal spectrum of $\Gamma$ decomposes into a direct Hilbert sum of closed irreducible subspaces $H_{\pi}$ with finite multiplicities. This leads to a decomposition of $H_{\text {cusp }}^{*}(X / \Gamma, E)$ into a finite algebraic sum of cohomological spaces. This sum is parametrized by the set of equivalence classes of irreducible unitary representations of $G(\mathbb{R})$ whose infinitesimal character coincides with the one of the contragradient representation $E^{*}$ of $E$. Thus, cuspidal cohomology isolates a finite set (depending on $E$ ) of representations occuring in the cuspidal spectrum.

For example, if $G=S L_{2} / \mathbb{Q}, \Gamma \subset S L_{2}(\mathbb{Z})$ and $E=E_{k}$ has dimension $k$, then via the Eichler-Shimura isomorphism

$$
H_{\text {cusp }}^{1}\left(X / \Gamma, E_{k}\right) \cong S_{k+1}^{+}(\Gamma) \oplus S_{k+1}^{-}(\Gamma)
$$

one has an identification of the cuspidal cohomology $H_{c u s p}^{1}\left(X / \Gamma, E_{k}\right)$ with the spaces of holomorphic and anti-holomorphic cuspidal automorphic forms with respect to $\Gamma$ of weight $k+1$. The dimension of $S_{k+1}^{ \pm}(\Gamma)$ is the multiplicity with which the holomorphic (antiholomorphic) discrete series representation $D_{k}^{ \pm}$of $S L_{2}(\mathbb{R})$ occurs in the cuspidal spectrum.

It is a major issue to prove the existence of cuspidal automorphic forms which give rise to non-vanishing cuspidal cohomology classes. It is even more challenging if the real Lie group $G(\mathbb{R})$ does not have discrete series representations. This is the case if the real rank of $G(\mathbb{R})$ is not equal to the rank of a maximal compact subgroup $K$ of $G(\mathbb{R})$. For example, this is the case for the group $P G L_{2}(\mathbb{C})$ we are interested in. We refer to [28] $\S 5$ for a survey of results by which one can detect cuspidal cohomology classes also in those cases.

4.2. Base change for $G L(2)$. Let $F$ be an algebraic number field, and denote by $A\left(G L_{2}, F\right)$ the set of equivalence classes of irreducible automorphic representations of $G L_{2}\left(\mathbb{A}_{F}\right)$. Let $A_{0}\left(G L_{2}, F\right)$ be the subset of $A\left(G L_{2}, F\right)$ of equivalence classes of irreducible cuspidal automorphic representations of $G L_{2}\left(\mathbb{A}_{F}\right)$. Given an extension $E / F$ of number fields, and an automorphic representation $\pi,[\pi] \in A\left(G L_{2}, F\right)$, the principle of Langlands' functoriality predicts that there should be an automorphic representation $\pi^{\prime}$ of $G L_{2}\left(\mathbb{A}_{E}\right)$ canonically associated with $\pi$ called the base change lift. There are two cases in which this lifting (for the group $G L_{2}$ ) is well understood.

(1) If $E / F$ is a cyclic extension of prime degree, the existence of a lift has been proved by Langlands [26], using the twisted trace formula. [Notice that a solvable extension may be built up by a series of cyclic extensions]. The trace formula approach gives a precise characterization of the image (resp. the fibres) of the lift.

(2) If $E / F$ is a cubic extension, even possibly not normal, the existence of $\pi^{\prime}$ was proved by Jacquet et al. [23] using the converse theorem.

Thus, there are maps

$$
\Psi_{(i)}: A\left(G L_{2}, F\right) \longrightarrow A\left(G L_{2}, E\right), i=1,2
$$

in the two cases just described. If $E / F$ is an extension so that there is a tower $E=F_{m} \supset F_{m-1} \supset \ldots \supset F_{0}=F$ of intermediate fields so that $F_{j+1} / F_{j}$ 
is of the form discussed in (1) and (2) respectively there is an analogous map $\Psi: A\left(G L_{2}, F\right) \longrightarrow A\left(G L_{2}, E\right)$ as well; it is defined as the compositum of maps $\Psi_{(i)}$ as necessary. One of the critical properties of this map is the following (cf. [25] Prop. 4.5.): If $\pi$ is a non-dihedral cuspidal automorphic representation, then its base change lift $\Psi(\pi)$ is a cuspidal representation of $G L_{2}\left(\mathbb{A}_{E}\right)$. In particular, this correspondence permits to associate to a cuspidal representation $\pi$ of $G L_{2}\left(\mathbb{A}_{F}\right)$ subject to the local condition of being special at a finite number of places $v \in V_{f}$ a cuspidal representation $\Psi(\pi)$ of $G L_{2}\left(\mathbb{A}_{E}\right)$ whose local component at any place of $E$ above $v$ is again of this type. The map is compatible (in a sense made precise in [25], §5) with cohomology. In particular, if $F$ is a totally real field, suppose that the archimedean component of $\pi$ is a discrete series representation (i.e. it also has non-vanishing relative Lie algebra cohomology with respect to some coefficient system $V$ ) then the archimedean component of $\Psi(\pi)$ has non-vanishing relative Lie algebra cohomology with respect to a suitable coefficient system only depending on $V$. For example, if the local component $\pi_{v}, v \in V_{\infty}$, is a discrete series representation of lowest $K$-type 2 (here we have $V=\mathbb{C}$ to obtain non-vanishing cohomology) then the local component of $\Psi(\pi)$ at an archimedean place has non-vanishing cohomology with respect to the trivial coefficient system as well.

4.3. Non-vanishing cuspidal cohomology. To describe more precisely the cohomological consequences of the base change lift for $G L_{2}$, one has to enlarge the framework in which the cohomology of arithmetic groups is considered, as it is the case in the theory of automorphic forms. Setting aside the fine points of this approach in terms of algebraic groups over the adèles the main application of the correspondence defined by base change can be formulated as follows (cf. [25])

TheOREm. Let $E / F$ be a finite extension of a totally real algebraic number field $F$ so that there is a tower

$$
E=F_{m} \supset F_{m-1} \supset \ldots \supset F_{0}=F
$$

of intermediate fields such that $F_{j+1} / F_{j}$ is cyclic of prime degree or a cubic nonnormal extension. Then there exists a congruence subgroup $\Gamma \subset P G L_{2}\left(\mathcal{O}_{E}\right)$ of finite index such that the cuspidal cohomology of $\Gamma$

$$
H_{\text {cusp }}^{*}(X / \Gamma, \mathbb{C}) \neq 0 .
$$

In particular, if the number field $E$ has degree $d=r_{1}+2 r_{2}$ (where $r_{1}\left(\begin{array}{lll}\text { resp. } & \left.r_{2}\right)\end{array}\right)$ denotes the number of real (resp. complex) places then the cuspidal cohomology does not vanish in degree $r_{1}+r_{2}$.

The launch pad for this non-vanishing result is the fact that there always exist cuspidal automorphic representations of $G L_{2}\left(\mathbb{A}_{F}\right)$ whose archimedean component is a suitable discrete series representation and which is special at a given finite number of places $v \in V_{f}$, i.e., its local component is the Steinberg representation. This result ([25], 2.5) is obtained by inserting so called pseudo coeffecients in the Selberg trace formula.

Remarks. (1) Let $k=\mathbb{Q}(\sqrt{d})$ be an imaginary quadratic number field. Then this result serves as an alternative proof of the non-vanishing of the first cuspidal cohomology of Bianchi groups $\Gamma_{d}$ up to commensurability. Notice that the first and 
the second cuspidal cohomology are isomorphic in this case. We refer to [16], [17] and [44] for more precise results.

(2) Recall that a solvable extension $E / F$ may be built up by a series of cyclic extensions. Hence, the description of the image and the fibres of the base change lift in [39] can be used to extend the result above to the case of an extension $E / F$ of a totally real number field $F$ where $E$ is contained in a solvable extension of $F$ (cf. $[40])$.

4.4. Jacquet-Langlands correspondence. Let $E / F$ be a finite extension of a totally real algebraic number field as in 4.3 . Suppose that $E$ has exactly one complex place and an arbitrary non-empty set $T$ of real places. Let $G^{\prime} / E$ be an inner form of the algebraic group $G / E=P G L_{2} / E$ defined over $E$ so that the derived group of $G^{\prime}$ is of the type $S L_{1}(D)$ where $D$ is a division quaternion algebra defined over $E$ which ramifies at all real places $v \in T$. Recall that at a place $v \in V$ where $D$ does not ramify one has an isomorphism

$$
G^{\prime}\left(E_{v}\right) \sim\left(E_{v}\right) \cong P G L_{2}\left(E_{v}\right), v \notin \operatorname{Ram}(D) .
$$

In particular, if $w$ denotes the unique complex place of $E$, one has

$$
G^{\prime}\left(E_{w}\right) \stackrel{\sim}{\rightarrow} P G L_{2}(\mathbb{C}) .
$$

On the other hand, since $T \subset \operatorname{Ram}(D)$, the group $G^{\prime}\left(E_{v}\right)$ is compact at a real place $v \in T$. Notice that the groups $G^{\prime}$ and $G$ are isomorphic over $E_{v}$ for almost all $v \in V$ because the ramification set $\operatorname{Ram}(D)$ is finite. This latter set (where $G^{\prime}$ is non-trivially twisted) will be denoted by $S$.

Given a torsion free arithmetic subgroup $\Gamma$ of $G^{\prime}(E)$ the corresponding hyperbolic 3 -manifold $H^{3} / \Gamma$ is compact.

Using the comparison between the trace formula for $G$ and $G^{\prime}$ and some local results Jacquet and Langlands have established a correspondence between automorphic representations of $G$ and $G^{\prime}$ [22]. In particular, starting off from a cuspidal automorphic representation $\pi$ of $G$ over $E$ as constructed in 4.3. (that is, whose archimedean components $\pi_{v}, v \in S \cap V_{\infty}$, are discrete series representations and whose local components $\pi_{v}, v \in S \cap V_{f}$ a finite place of $E$, are the Steinberg representation) there is a corresponding representation $\pi^{\prime}$ of $G^{\prime}$ over $E$ so that $\pi_{v}^{\prime}=\pi_{v}$ for $v \notin S$. Moreover, the representation $\pi^{\prime}$ contributes non-trivially to the automorphic spectrum of $G^{\prime}$. Notice that the local archimedean component $\pi_{w}, w$ the unique complex place of $E$, has non-trival cohomology with respect to trivial coefficients. As a consequence, one has a certain analogue of the Theorem in 4.3. in the compact case.

4.5. Theorem. Let $E / F$ be a finite extension of a totally real algebraic number field $F$ so that there is a tower

$$
E=F_{m} \supset F_{m-1} \supset \ldots \supset F_{0}=F
$$

of intermediate fields such that $F_{j+1} / F_{j}$ is cyclic of prime degree or a cubic nonnormal extension. Suppose that $E$ has exactly one complex place and an arbitrary non-empty set $T$ of real places. Let $G^{\prime} / E$ be an inner form of $G / E=P G L_{2} / E$, so that the finite set $S$ of places $v \in V$ where $G^{\prime}\left(E_{v}\right)$ is not isomorphic to $G\left(E_{v}\right)$ contains $T$. Then the following holds: Given an arithmetic congruence subgroup $\Gamma$ in $G^{\prime}(E)$ there exists a subgroup $\Gamma^{\prime}$ of $\Gamma$ of finite index so that the first cohomology $H^{1}\left(H^{3} / \Gamma^{\prime}, \mathbb{C}\right)$ of the compact hyperbolic 3-manifold $H^{3} / \Gamma^{\prime}$ does not vanish. 
4.6. Remarks and examples. (1) This result and its method of proof (by means of the theory of automorphic forms) provides an alternative approach to the non-vanishing assertion for the first Betti number of arithmetically defined cocompact Kleinian groups dealt with in Theorem 3.3. There they are interpreted as groups of units (up to commensurability) of suitable quadratic forms over $F$. More precisely, by 1.6., this class of cocompact Kleinian groups corresponds to arithmetic subgroups in an inner form $G^{\prime} / E$ where the underlying quaternion division algebra $D$ is of the form $B \otimes_{F} E, E$ a quadratic extension (with exactly one complex place) of $F$ and $B$ a quaternion division algebra over $F$ which ramifies at all real places not corresponding to the inclusion $F \longrightarrow \mathbb{R}$. Thus, 4.5. contains this non-vanishing result for the first Betti number $b_{1}\left(H^{3} / \Gamma\right), \Gamma$ as in 3.3. (with $n=3$ ), as a special case.

(2) Example (1) can be looked at in a more conceptual frame work. Let $E$ be an algebraic number field which has exactly one complex place and an arbitrary nonempty set $T$ of real places, let $G^{\prime} / E$ be an inner form of $G / E=P G L_{2} / E$, so that the finite set $S$ of places $v \in V$ where $G^{\prime}\left(E_{v}\right)$ is not isomorphic to $G\left(E_{v}\right)$ contains $T$. Suppose that the defining field $E$ has a subfield $F$ of index two. A proper subfield of $E$ has to be a totally real field due to the properties of the set of places of $E$. The extension $E / F$ (of degree 2 ) is a cyclic Galois extension. Thus, we are in the situation of 4.5 .

Let $\operatorname{Gal}(E / F)=\left\{I d_{E}, c\right\}$ denote its Galois group. Given a central simple Ealgebra $A$ its conjugate algebra $A^{c}=\left\{a^{c} \mid a \in A\right\}$ is defined by the following operations:

$$
a^{c}+b^{c}=(a+b)^{c}, a^{c} \cdot b^{c}=(a b)^{c},(\lambda a)^{c}=c(\lambda) a^{c}
$$

for all $a, b \in A$ and $\lambda \in E$. The map

$$
s: A^{c} \otimes_{E} A \rightarrow A^{c} \otimes_{E} A
$$

defined by $a^{c} \otimes b \mapsto b^{c} \otimes a$ is $c$-semilinear over $E$ and is an $F$-algebra automorphism. The $F$-subalgebra

$$
\left\{z \in A^{c} \otimes_{E} A \mid s(z)=z\right\}=: N_{E / F}(A)
$$

of elements in $A^{c} \otimes_{E} A$ fixed under $s$ is called the norm of the central simple algebra $A$ over $E$. It is a central simple $F$-algebra of degree $\operatorname{deg} N_{E / F}(A)=(\operatorname{deg} A)^{2}$. The norm induces a group homomorphism of Brauer groups (cf. [24], 3.13)

$$
N_{E / F}: \operatorname{Br}(E) \rightarrow \operatorname{Br}(F) .
$$

If $A=D$ is the quaternion division algebra underlying the given inner form $G^{\prime} / E$ of $G / E$ then its equivalence class in the Brauer group $\operatorname{Br}(E)$ is of order 2. Thus, the norm $N_{E / F}(D)$ of the $E$-algebra $D$ has order 1 or 2 in $\operatorname{Br}(F)$. Recall that the unit element in this group is the class of $F$ which is also the class of all the matrix algebras over $F$.

Suppose that $N_{E / F}(D)$ has order 1 . As an algebra of degree 4 over $F N_{E / F}(D)$ is isomorphic to the matrix algebra $M_{4}(F)$, that is, it splits. By a result of Albert und Riehm ([24], 3.1.), $N_{E / F}(D)$ splits if and only if there is an involution of the second kind on $D$ which fixes $F$ elementwise. Let $\tau$ denote this involution of the second kind. By definition of this notion, the restriction of $\tau$ to the center of $D$ is of order 2 , hence $\tau_{\mid E}$ coincides with $c$. As Albert has proved (cf [24], 2.22), an involution of the second 
kind on a quaternion algebra has a particular type. There exists a unique quaternion $F$-subalgebra $D_{0} \subset D$ such that $D=D_{0} \otimes_{F} E$ and $\tau$ is of the form $\tau=\gamma_{0} \otimes c$ where $\gamma_{0}$ is the canonical involution (also called quaternion conjugation) on $D_{0}$. The algebra $D_{0}$ is uniquely determined by these conditions. As it turns out, this case is the one dealt with in example (1).

Suppose that $N_{E / F}(D)$ has order 2 in the Brauer group $\operatorname{Br}(F)$. Then it is of the form $M_{2}(Q)$ where $Q$ is a quaternion division algebra over $F$. These division algebras $D$ (and thus the corresponding inner forms) give rise to the family of cocompact arithmetic groups in $\mathrm{SO}_{0}(3,1)$ associated to skew Hermitian forms over quaternion division algebras. Notice that, by the result of Albert and Riehm, the corresponding 3-dimensional hyperbolic manifolds do not admit totally geodesic hypersurfaces. This class of cocompact arithmetic Kleinian groups, commensurable to groups of units of skew-Hermitian forms in quaternionic vector spaces, was considered in [27]. Li and Millson obtain a non-vanishing result for the first Betti number in this case by analyzing a quaternionic theta series and an associated period integral.

However, the following implication of Theorem 4.5. gives a unified approach to the non-vanishing result in the case of the two classes just considered.

4.7. Theorem. Let $H^{3} / \Gamma$ be an arithmetically defined hyperbolic 3-manifold where $\Gamma$ is a congruence group. Suppose that the defining field $k$ has a subfield of index two. Then there exists a finite covering of $H^{3} / \Gamma$ with non-vanishing first Betti number.

Proof. The defining field $k$ has exactly one complex place and an arbitrary (but possibly empty) set of real places. If $\Gamma$ is not cocompact, then $k$ is an imaginary quadratic field. In this case the assertion is proved in 3.6. If $\Gamma$ is cocompact, a proper subfield $l$ of $k$ has to be a totally real field due to the number of places of $k$. Then 4.5. applies.

4.8. Cubic non-normal extensions. Let $k$ be an algebraic number field of degree $n=r_{1}+2 r_{2}$. The sign of its discriminant is determined by the number of complex places of $k$, that is, one has $\operatorname{sign}\left(d_{k}\right)=(-1)^{r_{2}}$. Thus, given a cubic extension $E / \mathbb{Q}$ which has exactly one complex place its discriminant $d_{E}$ is negative. Such a field is necessarily non-normal over $\mathbb{Q}$. More precisely, if $E=\mathbb{Q}(x)$, its normal closure $N$ is a quadratic extension of $E$. It can be described as $N=E\left(\sqrt{d_{E}}\right)_{i}$ its Galois group $G(N / \mathbb{Q})$ is isomorphic to $S_{3}$, the symmetric group in three letters.

By use of Cardan's formula for the root of a cubic polynomial $X^{3}+a X^{2}+b X+c$ over $\mathbb{Q}$ such cubic non-normal extensions can be easily constructed. Notice that any cubic can be reduced to the form $g=X^{3}+p X+q$ by a change of variable. If the discriminant $-4 p^{3}-27 q^{2}$ of $g$ is negative than $g$ has a unique real root. Adjoining a root of $g$ to $\mathbb{Q}$ gives a cubic extension $E$ of the desired type. For example, let $E=\mathbb{Q}(x)$ where $x$ is a root of the cubic polynomial $x^{3}-x-1$. This is the unique cubic field of discriminant -23 .

Let $D$ be a division quaternion algebra over $E$ which ramifies at least at the real place (and one finite place) of $E$. Then the corresponding compact hyperbolic 3-manifolds have non-vanishing first Betti number up to covering.

4.9. Virtual positive Betti number conjecture. One of the most interesting conjectures in 3-manifold theory is the one by Waldhausen [57] stated in 1968. It says: 
Suppose $M$ is an irreducible 3 -manifold whose fundamental group is infinite. Then there exists a finite covering $M^{\prime}$ of $M$ which is Haken, that is, it is irreducible and contains an embedded incompressible surface. An even stronger form states (under the same assumptions) that there exists a finite covering $M^{\prime}$ with non-vanishing first Betti number $b_{1}\left(M^{\prime}\right)$. This form is called the virtual positive Betti number conjecture and usually attributed to Thurston ([8], 1.2.). The significance of the former conjecture lies in the fact that it is known that 3-manifolds which are virtually Haken are geometrizable.

As the most challenging one, the case of hyperbolic 3-manifolds has gained increasing attention in recent years. Some results confirming the conjecture in specific cases were obtained in [34], [30], by geometric techniques (cf. our section 3) and in [25], [6] by an automorphic approach. Further evidence is given by the experiments described in [8]. For the analogous question in the case of hyperbolic $n$-manifolds we refer to [34], [27], [58].

Theorems 4.5 and 4.7. provide examples where this conjecture is proved. As a consequence of the discussion in 4.8. one obtains

THEOREM Let $H^{3} / \Gamma$ be an arithmetically defined hyperbolic 3-manifold where $\Gamma$ is a congruence group. Suppose that the defining field $k$ is a cubic non-normal extension of $\mathbb{Q}$. Then there exists a finite covering of $H^{3} / \Gamma$ with non-vanishing first Betti number.

Nonetheless, the original conjecture remains open in a number of cases. For example, let $E=\mathbb{Q}(x)$ where $x$ is a root of the irreducible quintic polynomial $g=$ $X^{5}-9 X+3$ over $\mathbb{Q}$ (or take $f=X^{5}-16 X+2$ ). The polynomial has three real roots and two conjugate complex roots. The extension $E / \mathbb{Q}$ has degree 5 and is nonnormal. It is not contained in any solvable extension. Let $D$ be a division quaternion algebra over $E$ which ramifies at least at the three real places (and one finite place) of $E$. Given an arithmetic subgroup in the units of $D$ the virtual positive Betti number conjecture is not known to be true in this case at hand. To my knowledge the methods known so far do not apply. Any progress in the base change construction as described above improves the situation.

\section{REFERENCES}

[1] R.C. Alperin, An elementary account of Selberg's lemma, Enseign Math., 33 (1987), pp. 269273.

[2] L. BIANchi, Sui gruppi di sostitutioni lineari con coefficienti a corpi quadratici imaginari, Math. Annalen, 40 (1892), pp. 332-411.

[3] A. Borel, Introduction aux Groupes Arithmétiques, Hermann, Paris, 1969.

[4] A. Borel, Commensurability classes and volumes of hyperbolic 3-manifolds. Ann. Scuola Norm. Sup. Pisa, 8 (1981), pp. 1-33.

[5] A. Borel, J.-P. LABesse, J. Schwermer, On the cuspidal cohomology of S-arithmetic groups of reductive groups over number fields. Compositio Math., 102 (1996), pp. 1-40.

[6] L. ClOzEL, On the cuspidal cohomology of arithmetic subgroups of $S L(2 n)$ and the first Betti number of arithmetic 3-manifolds. Duke Math. J., 55 (1987), pp. 475-486.

[7] A. Dold, Lectures on Algebraic Topology (Grundlehren Math. Wiss. Bd. 200) BerlinHeidelberg-New York: Springer 1972.

[8] N. Dunfield And W.P. Thurston, The virtual Haken conjecture: experiments and examples. Geometric Topol., 7 (2003), pp. 399-441.

[9] J. Elstrodt, F. Grunewald, J. Mennicke, Groups acting on hyperbolic space. BerlinHeidelberg-New York: Springer 1997. 
[10] J. Franke, Harmonic analysis in weighted $L_{2}$-spaces, Ann. Sci. Ecole Norm. Sup. (4) 31 (1998), no. 2, pp. 181-279.

[11] J. Franke And J. Schwermer, A decomposition of spaces of automorphic forms, and the Eisenstein cohomology of arithmetic groups, Math. Ann., 311:4 (1998), pp. 765-790.

[12] M. Gromov and I. Piatetski-Shapiro, Non-arithmetic groups in Lobachevsky spaces, Publ. Math., Inst. Hautes Etudes Sci., 66 (1988), pp. 93-103.

[13] F. GRunewald And S. KüHnlein, On the proof of Humbert's volume formula, Manuscripta Math., 95 (1998), pp. 431-436.

[14] F. Grunewald And J. Schwermer, Arithmetic quotients of hyperbolic 3-space, cusp forms and link complements. Duke Math. J., 48 (1981), pp. 351-358.

[15] F. Grunewald and J. Schwermer, Free non-abelian quotients of $\mathrm{SL}_{2}$ over orders of imaginary quadratic number fields, J. Algebra, 69 (1981), pp. 298-304.

[16] F. Grunewald And J. Schwermer, A non-vanishing theorem for the cuspidal cohomology of $S L_{2}$ over imaginary quadratic integers, Math. Annalen, 258 (1981), pp. 183-200.

[17] G. HARder, On the cohomology of $S L(2, \mathcal{O})$. In: Lie groups and their representations. Proc. of the Summer School on Group Representations, pp. 139-150. London: Hilger 1975.

[18] G. HARDER, On the cohomology of discrete arithmetically defined groups, Discrete subgroups of Lie groups and applications to moduli (Internat. Colloq., Bombay, 1973), pp. 129-160. Oxford Univ. Press, Bombay, 1975.

[19] G. HaRder, Eisenstein cohomology of arithmetic groups. The case $G L_{2}$, Invent. Math., 89:1 (1987), pp. 37-118.

[20] G. HARDER, A Gauss-Bonnet formula for discrete arithmetically defined groups, Ann. Sci. Ec. Norm. Sup. (4) 4 (1971), pp. 409-455.

[21] S. Helgason, Differential Geometry and Symmetric Spaces, Academic Press, New York and London, 1962.

[22] H. JacQuet and R.P. Langlands, Automorphic Forms on GL(2), Lect. Notes in Math., 114, Berlin-Heidelberg-New York: Springer 1970.

[23] H. Jacquet, I. Piatetski-Shapiro, J. Shalika, Relevement cubique non normal. C.R. Acad. Sci. Paris, 292 (1981), pp. 567-571.

[24] M.-A. Knus, A. Merkurjev, M. Rost And J.-P. Tignol, The Book of Involutions. Colloquium Publications 44. AMS 1998.

[25] J.P. LABEsSe AND J. SChwermer, On liftings and cusp cohomology of arithmetic groups, Invent. Math., 83 (1986), pp. 383-401.

[26] R.P. Langlands, Base Change for GL(2), Ann. of Math. Studies, vol. 96, Princeton: Princeton Univ. Press 1980.

[27] J.S. Li AND J.J. MilLson, On the first Betti number of a hyperbolic manifold with an arithmetic fundamental group, Duke Math. J., 71 (1993), pp. 365-401.

[28] J.S. Li AND J. SchWermer, Automorphic representations and cohomology of arithmetic groups, "Challenges for the 21st century (Singapore, 2000), pp. 102-137, World Sci. Publishing, 2001.

[29] J.S. Li And J. Schwermer, On the Eisenstein cohomology of arithmetic groups, Duke Math. J., 123 (2004), pp. 141-169.

[30] A. Lubotzky, Free quotients and the first Betti number of some hyperbolic manifolds, Transformation groups, 1 (1996), pp. 71-82.

[31] C. Maclachlan and A. Reid, The Arithmetic of Hyperbolic 3-manifolds, Graduate Texts in Maths. 219, Springer: New York, Berlin, Heidelberg 2003.

[32] G.A. Margulis and E. Vinberg, Some linear groups virtually having a free quotient, J. Lie theory, 10 (2000), pp. 171-180.

[33] E. Mendoza, Cohomology of $P G L_{2}$ over imaginary quadratic integers, Bonner Math. Schriften 128. Math. Institut, Universität Bonn, Bonn 1980

[34] J. Millson, On the first Betti number of an hyperbolic manifold, Annals Maths., 104 (1976), pp. 235-247.

[35] J. Millson and M.S. Raghunathan, Geometric construction of cohomology for arithmetic groups I. In: Geometry and Analysis (Papers dedicated to the memory of Patodi), pp. 103-123, Indian Academy of Sciences, Bangalore 1980.

[36] J. Milnor and J. Stasheff, Characteristic classes, Ann. of Math. Studies, vol.76, Princeton: Princeton Univ. Press 1974.

[37] M.S. Raghunathan, The first Betti number of compact locally symmetric spaces. In: Current Trends in Mathematics and Physics. 116-137, New Delhi: Narosa 1995.

[38] M.S. Raghunathan and T.N. Venkataramana, The first Betti number of arithmetic groups and the congruence subgroup problem, in: Linear Algebraic Groups and their Representations, Contemporary Maths. 153 (1993), pp.95-107. 
[39] C.S. RAJAn, On the image and fibres of solvable base change, Math. Res. Letters, 9 (2002), pp. 499-508.

[40] C.S. RAJAN, On the non-vanishing of the first Betti number of hyperbolic three manifolds, preprint 2003.

[41] J.G. Ratcliffe, Foundations of Hyperbolic Manifolds, Graduate Texts Maths. 149. New YorkBerlin-Heidelberg: Springer 1994.

[42] I. Reiner, Maximal orders, London-New York: Academic Press 1975.

[43] J. Rohlfs, Arithmetisch definierte Gruppen mit Galoisoperation, Invent. Math., 48 (1978), pp. 185-205.

[44] J. Rohlfs, On the cuspidal cohomology of the Bianchi modular groups, Math. Zeitschrift, 188 (1985), pp. 253-269.

[45] J. Rohlfs And J. Schwermer, Intersection numbers of special cycles, J. American Math. Soc., 6 (1993), pp. 755-778.

[46] J. Rohlfs And J. Schwermer, An arithmetic formula for a topological invariant of Siegel modular varieties, Topology, 37 (1998), pp. 149-159.

[47] J. Schwermer, A note on link complements and arithmetic groups, Math. Annalen, 249 (1980), pp. 107-110.

[48] J. Schwermer, Kohomologie arithmetisch definierter Gruppen und Eisensteinreihen, Lect. Notes in Math., 988, Berlin-Heidelberg-New York: Springer 1983.

[49] J. Schwermer, Arithmetic Groups - Geometric Aspects, lectures at ETH Zürich (Nachdiplomvorlesung) 1999/2000, in preparation.

[50] J. Schwermer And K. Vogtmann, The integral homology of $S L_{2}$ and $P S L_{2}$ of euclidean imaginary quadratic integers, Comment. Math. Helvetici, 58 (1983), pp. 573-598.

[51] J-P. Serre, Corps Locaux. Paris: Hermann 1962.

[52] J-P. Serre, Cohomologie Galoisienne, Lect. Notes Maths. 5. Berlin-Heidelberg-New York: Springer 1964.

[53] J-P. Serre, Arbres, Amalgames, $S L_{2}$. Asterisque 42, Paris: Soc. Math. France: 1977.

[54] R. SwAN, Generators and relations for certain special linear groups, Advances Math., 6 (1971), pp. $1-77$.

[55] M.-F. Vigneras, Arithmetique des Algebres de Quaternions, Lect. Notes in Maths. 800. BerlinHeidelberg-New York: Springer 1980.

[56] K. Vogtmann, Rational homology of Bianchi groups, Math. Annalen, 272 (1985), pp. 399-419.

[57] F. Waldhausen, The word problem in fundamental groups of sufficiently large irreducible 3manifolds, Annals Maths., 88 (1968), pp. 272-280.

[58] X. XuE, On the Betti numbers of a hyperbolic manifold, Geometric and Functional Analysis, 2 (1992), pp. 126-136.

[59] H. Zassenhaus, On the units of orders, J. of Algebra, 20 (1972), pp. 368-395. 
